\title{
Efectividad de las técnicas de manejo conductual en odontopediatría. Revisión sistemática
}

\author{
Miriam Ortega, ${ }^{1}$ \\ María Tatiana Tapia Calderón, ${ }^{2}$ \\ Gabriela Estefanía Cedillo Mora, ${ }^{2}$ \\ Ronald Roossevelt Ramos Montiel, ${ }^{2}$ \\ Rita Mireya Navas Perozo. ${ }^{3}$
}

\section{Resumen}

Objetivo: Efectuar una revisión sistemática sobre la efectividad de las técnicas de manejo de la conducta en niños sistémicamente sanos, en la consulta de Odontopediatría. Métodos: Investigación descriptiva, retrospectiva, de estudios observacionales y ensayos clínicos aleatorios, controlados que evaluaron la efectividad de las técnicas en niños y adolescentes de 03 a 18 años de edad. Resultados: Los criterios de inclusión fueron artículos en inglés y español, publicados desde 2014 al 2019. El análisis de la calidad metodológica de los estudios se realizó mediante la guía Strobe y CASPe. Se identificaron términos de búsqueda en inglés en el tesauro MeSH en la base de datos PubM/Medline, Scopus /Elsevier, y en español, se utilizaron descriptores de las Ciencias de la Salud (DeCS/BIREME) en la base de datos SciELO.org Redalyc.org, Latindex, Dialnet y google académico.
Hubo homogeneidad en los estudios respecto a la selección de escalas para medir ansiedad y miedo en los niños al recibir tratamiento preventivo, curativo o restaurador. Los estudios evaluaron mayoritariamente técnicas de distracción aplicadas con recursos audiovisuales para determinar su efectividad sobre el comportamiento de los niños en el consultorio odontológico. También reportaron disminución de la ansiedad y miedo, después de la aplicación de las técnicas de distracción, e informaron que hubo cambios de conducta de pacientes ansiosos y negativos a pacientes positivos y colaboradores. Conclusiones: Los estudios mostraron una insuficiente calidad en sus diseños, lo que no permitió emitir juicios respecto a la contundencia y solidez de la evidencia sobre la efectividad de las técnicas aplicadas.

Palabras clave: DeCS: efectividad, técnicas, conducta, niños, odontopediatria.

\footnotetext{
${ }^{1}$ Universidad UTE.

${ }^{2}$ Universidad Católica de Cuenca.

${ }^{3}$ Universidad del Zulia.
} 
Artigo da revisao

\section{Eficácia das técnicas de gestão comportamental na odontologia pediátrica. Revisão sistemática}

\section{Resumo}

Objetivo: Realizar uma revisão sistemática da eficácia das técnicas de gerenciamento de comportamento em crianças sistemicamente saudáveis, na consultório de Odontopediatria. Métodos: Estudos descritivos, retrospectivos, observacionais e ensaios clínicos controlados e randomizados que avaliaram a eficácia das técnicas em crianças e adolescentes de 03 a 18 anos. Resultados: Os critérios de inclusão foram artigos em inglês e espanhol, publicados de 2014 a 2019. A análise da qualidade metodológica dos estudos foi realizada na guia Strobe and Consort. Os termos de pesquisa em inglês foram identificados na tesauro MeSH na base de dados PubMed / Medline, Scopus / Elsevier e, em espanhol, os descritores de Ciências da Saúde (DeCS / BIREME) foram utilizados na base de dados SciELO.org Redalyc.org, Latindex, Dialnet e Google Scholar. Houve homogeneidade nos estudos em relação à seleção de escalas para medir ansiedade e medo em crianças ao receber tratamento preventivo, curativo ou restaurador. Os estudos avaliaram principalmente técnicas de distração aplicadas com recursos audiovisuais para determinar sua eficácia na comportamento de crianças na consultório odontológico. Eles também relataram diminuição da ansiedade e medo, após a aplicação de técnicas de distração, e relataram que houve mudanças comportamentais de pacientes ansiosos e negativos para pacientes positivos e colaborativos. Conclusões: Os estudos mostraram qualidade insuficiente em seus desenhos, o que não permitiu julgamentos sobre a força e a força das evidências sobre a eficácia das técnicas aplicadas.

Palavras-chave: DeCS: efetividade, técnicas, comportamento, crianças, odontologia pediátrica.

Article of revision

\section{Effectiveness of behavioral management techniques in pediatric dentistry. Systematic review}

\begin{abstract}
Objective: To conduct a systematic review of the effectiveness of behavior management techniques in systemically healthy children, in the Pediatric Dentistry office. Methods: Descriptive, retrospective, observational studies and randomized controlled clinical trials
\end{abstract}

that evaluated the effectiveness of the techniques in children and teenangers aged 03 to 18 years. Results: The inclusion criteria were articles in English and Spanish, published from 2014 to 2019. The analysis of the methodological quality of the studies was carried out using the Strobe and Consort guide. English search terms were identified 
in the MeSH thesaurus in the PubM / Medline, Scopus / Elsevier database, and in Spanish, descriptors of Health Sciences (DeCS / BIREME) were used in the SciELO.org database, Redalyc.org, Latindex, Dialnet and Google Scholar. There was homogeneity in the studies regarding the selection of scales to measure anxiety and fear in children when receiving preventive, curative or restorative treatment. The studies mostly evaluated distraction techniques applied with audiovisual resources to determine their effectiveness on the behavior of children in the dentistry office. They also

\section{Introducción}

La prevalencia de la caries dental en niños escolares, según la Organización Mundial de la salud (OMS), oscila entre $60-90 \%$ a nivel mundial ${ }^{1}$ por lo cual es considerada un problema de salud pública. La Federación dental internacional (FDI $)^{2}$ afirma que la carga de esta enfermedad a los 12 años es mayor en los países con ingresos moderados en los que aproximadamente las $2 / 3$ partes de las caries no son tratadas, incluso en los países con alto nivel de ingresos, más de la mitad de las caries permanecen sin tratamiento.

Según esta Federación la caries no tratada puede dificultar comer y dormir, puede tener impacto en el crecimiento del niño y es la causa principal de ausentismo escolar. Por ser multifactorial, existe un amplio rango de factores que influyen y determinan la presencia de caries y su severidad entre ellas las condiciones socioeconómicas ${ }^{3-5}$, los aspectos culturales ${ }^{6}$, la educación ${ }^{4}$, el estilo de vida ${ }^{7}$, así como las prácticas y cuidados de salud. ${ }^{2}$ reported decreased anxiety and fear, after the application of distraction techniques, and reported that there were behavioral changes from anxious and negative patients to positive and collaborative patients. Conclusions: The studies showed insufficient quality in their designs, which did not allow judgments regarding the strength and strength of the evidence on the effectiveness of the techniques applied.

Key words: Effectiveness, techniques, Behavior, Child, Pediatric Dentistry.

La Academia Americana de Odontología Pediátrica $(\mathrm{AAOP})^{8}$ en conjunto con la Academia Americana de Pediatría (AAP) ${ }^{9}$ señalan que los médicos de atención primaria deben referir a los niños, basados en la evaluación de riesgo, a la consulta odontológica a partir de los 06 meses y no más allá de los 12 meses de edad. Plantea que el ejercicio de la odontología pediátrica, no puede ni debe limitarse solo a la prevención y solución de los problemas bucales, sino que lleva implícito también el desempeño de un papel importante en los sectores psicológico y educacional donde la relación humana entre odontólogo, niño y padres es fundamental para el éxito del tratamiento frente a diversas situaciones ${ }^{8}$.

Una de esas situaciones es la que se refiere a las fobias dentales, la ansiedad y el miedo ${ }^{10}$. Ello ha sido reconocido como una fuente de problemas en el manejo del paciente pues influye en un comportamiento no cooperador, el cual podría impedir un tratamiento dental oportuno ${ }^{11}$ Soler y $\mathrm{col}^{12}$ definen la ansiedad como "aquel sentimiento vital, consciente, que sitúa al 
organismo en alerta ante la previsión de un peligro próximo que puede amenazar su integridad física o psíquica. Puede ser sustituto psicológico del dolor y se puede anticipar a él, mediante la experiencia, memoria y procesos asociativos. Resulta difícil muchas veces establecer una diferencia significativa entre ansiedad y miedo, siendo este último el que percibe mejor la causa del peligro.

Por otro lado, la ansiedad dental se ha descrito como el miedo a la consulta o al tratamientoodontológico,constituyéndose en una de las dificultades más frecuentes para el profesional, causando gran impacto en la salud oral ${ }^{13}$. Existen diversas causas que pueden dar lugar a la ansiedad en la consulta odontológica, entre ellas, la experimentación del dolor al tratamiento y a la causa del mismo, amenazas de los padres con la inyección del médico o del odontólogo si el niño se porta mal, antecedentes de experiencias negativas del entorno y problemas psicológicos de base entre otras ${ }^{13}$. Un estudio efectuado por Rodríguez et $a^{14}$ cuyo objetivo fue cuantificar el grado de ansiedad presente en pacientes infantiles y conocer los distintos factores que la producían utilizó la "encuesta de medida del miedo en niños, Subescala Dental (DS)" (Inventario de miedo para niños de Nakamura y Scherer (CFSS) y la "Escala de miedo dental de Kleinkecht. Sus resultados mostraron que los ítems con mayor puntuación en la Subescala Dental fueron los asociados a las inyecciones y el uso de las fresas en el consultorio dental.

Villasanta y Padilla ${ }^{11}$ estudiaron 152 niños y adolescentes en edades comprendidas entre 7 y 18 años, para identificar a los pacientes con conducta no cooperadora, frente al tratamiento dental, el estudio fue experimental y aplicaron la técnica de modelado con video preoperatorio. En el grupo experimental se reportó que los niños de 5 a 7 años de edad con conducta negativa ( $75 \%$ ) y definitivamente negativa (25\%), después de la aplicación del video preoperatorio modificaron su conducta a positiva $(60 \%)$ y definitivamente positiva (20\%) frente a la atención dental en una segunda sesión.

La colaboración del paciente es primordial para desarrollar un tratamiento. El grado de colaboración puede guardar relación con experiencias previas; por tanto, la manera como se atiende a un paciente, y en especial al paciente pediátrico, es crucial; debe considerase el componente biológico del niño como un sujeto en proceso de desarrollo tanto físico como psicológico, además de su contexto cultural y social. ${ }^{16}$

Por lo tanto el manejo de la conducta se considera un componente esencial en la práctica odontológica infantil, que tiene como objetivo establecer una buena comunicación con el paciente y ganarse su confianza, lo cual se logra a través del empleo de técnicas conductuales fundamentadas en la Psicología del Aprendizaje destinadas a tales fines. Estas permiten ayudar a superar conductas difíciles y realizar de manera eficaz el tratamiento en el niño, instándole a mostrar una actitud dental positiva. ${ }^{17}$

La evaluación de la conducta del niño, previo a la consulta odontológica, es muy importante por cuanto ello permite discernir sobre cuáles serían las formas más apropiadas para su abordaje y en consecuencia ello garantiza el éxito de su intervención en la atención. Según Lazo et al ${ }^{18}$ la efectividad y adherencia a los tratamientos odontológicos en edades 
tempranas, dependen del comportamiento del niño en la consulta, por lo que se hace necesario reforzar y enseñar habilidades sociales para mejorar ese comportamiento y evitar posibles traumas en su relación con el odontólogo y que esto incida en sus conductas futuras para el mantenimiento de su salud bucal.

Ramos et al ${ }^{15}$ afirman que la atención odontológica en el niño no puede estar sujeta a un parámetro fijo o protocolo de manejo pre-establecido, ya que cada niño es diferente, con un tipo de conducta específica y a veces trastornos de comportamientos, los cuales deben ser identificados desde la primera consulta, con el fin de ser intervenidos en las próximas sesiones.

La Asociación Latinoamericana de Odontopediatría $(\mathrm{ALOP})^{19}$ señala que un odontólogo que trata a los niños debe conocer una variedad de técnicas de adaptación del comportamiento $\mathrm{y}$, en la mayoría de las situaciones, debe estar apto para evaluar exactamente el nivel de desarrollo del niño, sus actitudes, su temperamento, y predecir sus reacciones frente al tratamiento.

La Asociación Americana de Odontología Pediatica $^{8}$ sostiene en su documento "Guideline on behavior guidance for the pediatric dental patient", que los padres influyen en el comportamiento de sus hijos en el consultorio dental y sus actitudes positivas hacia la atención de la salud oral pueden conducir al establecimiento de cuidados preventivo tempranos, menos enfermedades bucales, disminución de necesidades de tratamiento y menos experiencias negativas.
Los padres que se sienten apoyados, se sentirán seguros sobre la salud bucal de sus hijos, y se infiere que tendrán un mejor cumplimiento de las recomendaciones médicas y odontológicas ${ }^{20}$, Por el contrario, padres con experiencias dentales negativa, pueden transmitir su propia ansiedad o miedo dental al niño, afectando negativamente la actitud $y$ respuesta del infante ${ }^{8}$.

Las razones que justifican una revisión sistemática sobre esta temática son diversas, entre ellas se pretende resumir y organizar un volumen extenso y disperso de literatura, que siguiendo los criterios metodológicos establecidos para las revisiones sistemáticas, proporcione una guía selectiva de un gran número de reportes de investigaciones científicas que es imposible de analizar en su totalidad y que además han tenido un acelerado desarrollo en los últimos 20 años en el mundo de la ciencia odontológica especialmente en Odontopediatría.

Conocer la magnitud de la efectividad de las técnicas de manejo de la conducta en niños sistémicamente sanos, en el consultorio odontológico, aportaría la mejor y más actualizada evidencia disponible sobre su efectividad, para que puedan ser utilizadas por los Odontopediatras, odontólogos y los responsables de las políticas de salud a fin de tomar decisiones sanitarias bien informadas.

\section{Objetivo}

Efectuar una revisión sistemática sobre la efectividad de las técnicas de manejo de la conducta en niños sistémicamente sanos, en la consulta odontopediatría. 


\section{Métodos}

Investigación de tipo descriptiva, retrospectiva, de la literatura de estudios primarios sobre la efectividad de las técnicas de manejo de conducta en Odontopediatría.

\section{Tipo de estudio seleccionado}

- Estudios experimentales, aleatorios, controlados, que evaluaron la efectividad de las técnicas no farmacológicas de manejo de la conducta en niños sistémicamente sanos, en el consultorio odontológico; comparado con una no intervención o con otras intervenciones o variaciones de la misma intervención.

- Estudios observacionales que indagaron la efectividad de las técnicas no farmacológicas de manejo de la conducta en niños sistémicamente sanos, en el consultorio odontológico.

Tipo de participantes

- Niños y adolescentes desde los 03 hasta los 18 años de edad, sistémicamente sanos, con o sin antecedentes de atención odontológica preventiva, curativa o rehabilitadora.

\section{Resultados}

- Intervenciones no farmacológicas sobre la efectividad de las técnicas de manejo de la conducta en el consultorio odontológico en niños y adolescentes desde 03 hasta los 18 años de edad, sistémicamente sanos.
Los criterios de exclusión de los estudios fueron: ensayos clínicos que utilizaron técnicas farmacológicas, que incluyeran niños con algún compromiso sistémico y estudios de cohortes, reporte de casos y revisiones.

El proceso de revisión abarcó los siguientes pasos

1. Definición de una pregunta de investigación para orientar el proceso de búsqueda (criterios PICO).

¿Cuál es la efectividad de las técnicas de manejo de la conducta en niños sistémicamente sanos, en la consulta odontopediátrica?

Formulación del plan de búsqueda de la literatura

Se establecieron como criterios de inclusión: artículos de investigaciones empíricas, en idioma inglés y español, publicados durante el periodo 2014 al 2019.

Se excluyeron artículos duplicados, es decir aquellos trabajos que aparecieron publicados en diferentes revistas, y artículos a los cuales no se tuvo acceso al texto completo.

Se identificaron los términos de búsqueda en el idioma inglés en el tesauro $\mathrm{MeSH}$ de la base de datos PubM/Medline, Scopus /Elsevier, sobre la efectividad de las técnicas del manejo de la conducta en niños en odontopediatría; estos términos fueron:

"Effectiveness behavior modification 
techniques in Pediatric Dental" OR "Anxious Dental Pediatric Patients", OR "dental fear Pediatric Patients" OR "restorative dental treatment in children"

1. "Efficacy of behavioral modification techniques in pediatric dentistry" OR "pediatric dental"

2. "Management of Anxious Dental in Pediatric Patients" OR "pediatric dental office"

3. "Effectiveness of active and passive distraction in pediatric dentistry"

En español, se utilizaron descriptores establecidos en el tesauro de las Ciencias de la Salud (DeCS/BIREME) en la base de datos SciELO.org Redalyc.org, Biblioteca Virtual en Salud/ BIREME, Latindex, Dialnet y google académico.

\section{Identificación, selección de los estudios}

La identificación y selección de los artículos se hizo de acuerdo a que los descriptores claves de los tesauros $\mathrm{MeSH}$ (ingles) y DeCS (español) aparecieran en el título, resumen y texto completo. Una vez identificados y seleccionados los artículos potencialmente elegibles, dos examinadores evaluaron de manera independiente los títulos y resúmenes obtenidos decidiendo su inclusión o exclusión, en los casos en los que se presentaron divergencias estas fueron resueltas por consenso.

Determinada la elegibilidad, los artículos se obtuvieronen textocompleto. Posteriormente, también de manera independiente, se les realizó el análisis de la calidad metodológica mediante la guía de calidad Strobe ${ }^{21}$ para estudios observacionales y CASPe para ensayos clínicos. ${ }^{22-23}$
Medline/PubMed, Scopus/Elsevier, SSciELO.org Redalyc.org; Medigraphic, Dialnet y Google Académico

Total: 52

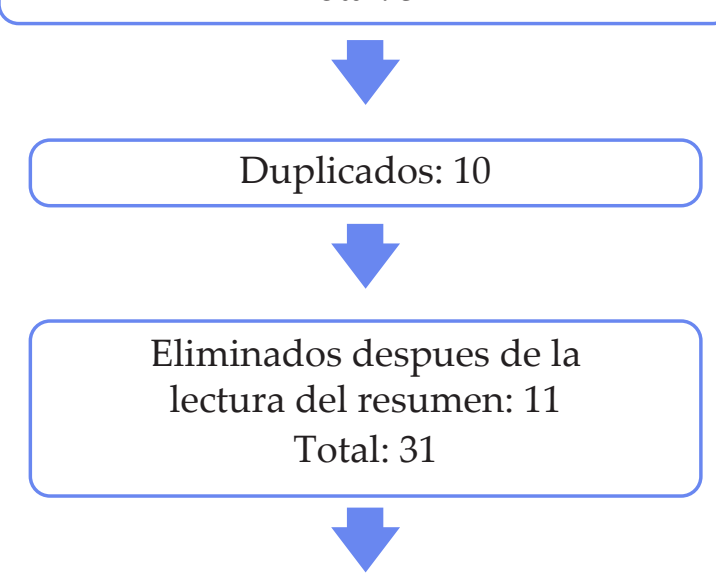

Eliminados después de la lectura crítica de los artículos completos: 18

\section{Total de artículos incluido para la} revisión: 13

Figura 1. Diagrama de búsqueda, filtro y selección de los artículos de la revisión de la Literatura.

La figura 1 ilustra el Diagrama del proceso de búsqueda, filtro y selección de los artículos de la revisión de la literatura.

\section{Resultados}

De los 13 artículos seleccionados para la revisión; 11 correspondieron a estudios experimentales aleatorios ${ }^{24-32,39}$ y 02 a estudios observacionales ${ }^{33,35}$ que evaluaron la efectividad de las técnicas de manejo de la conducta en niños sistémicamente sanos, en la consulta odontológica. Respecto al idioma, 09 
estaban publicados en inglés y 04 en español..$^{11,29,30,35}$ En relación al lugar de procedencia de las investigaciones, 06 procedían de la India, ${ }^{24,26,27,31,33,34} 01$ de España, 02 de Arabia Saudita, ${ }^{25,28} 02$ de Perú, ${ }^{11,30} 01$ de Ecuador, ${ }^{29} 01$ de México. ${ }^{32}$

Las edades de los niños fueron muy variables con un intervalo que comprendió entre los 04 hasta los 18 años y solo 1 estudio no informó las edades del niño participantes. ${ }^{25}$ El tamaño de la muestra en los estudios osciló entre los 3925 y 40035 niños, y su determinación fue variable $y$ en algunos casos no reportada.

En cuanto a la calidad metodológica de los estudios, al aplicar los criterios Strobe ${ }^{21}$ a las investigaciones observacionales, se encontraron bajos niveles de cumplimiento en lo referente a: describir los períodos de reclutamiento, exposición, seguimiento y recogida de datos, especificar todas las medidas adoptadas para afrontar fuentes potenciales de sesgo, explicar cómo se determinó el tamaño de la muestra e indicar las limitaciones de los estudios.

La calidad de los diseños de los estudios experimentales aleatorios, controlados fueron analizados mediante la aplicación de los criterios CASPe $\mathrm{C}^{22,23}$ entre los aspectos ausentes o escasamente reportados estaban: señalar quién asignó los participantes a la intervención; explicar el método de aleatorización utilizado, informar quién quedó ciego o enmascarado (pacientes o participantes, quien o quienes aplicaron la intervención o evaluaron los resultados), describir el proceso de la técnica de enmascaramiento utilizado. La mayoría de los estudios no reportaron si fue un solo Odontopediatra o varios quienes aplicaron y manejaron las escalas de medición de ansiedad, miedo, pulso, frecuencia cardiaca $y$ la evaluación de las técnicas o escalas de comportamiento, en las muestras seleccionadas, ni si los examinadores fueron previamente capacitados.

La tabla 1 muestra las características de los estudios incluidos en la revisión según identificación de sus autores, fuentes de publicación, título del trabajo, año, país y tamaño de la muestra utilizada.

Los resultados de los métodos empleados en los estudios de esta revisión se muestran en las tablas 2-1 y 2-2

Los Resultados de los hallazgos en los estudios de la revisión sobre Efectividad de las Técnicas de manejo conductual en Odontopediatría se resumen en las tablas 3-1, 3-2 y 3-3 
Tabla 1: Caracteristicas de los estudios incluidos en la revisión de la literatura Efectividad de las técnicas de manejo conductual en odontopediatría.

\begin{tabular}{|c|c|c|c|c|c|}
\hline$\overline{\text { Autores }}$ & Fuente & Titulo & Año & País & Muestra \\
\hline $\begin{array}{l}\text { 1. Singh D, } \\
\text { Samadi F, } \\
\text { Jaiswal JN, } \\
\text { Tripathi } \mathrm{AM}^{24}\end{array}$ & $\begin{array}{l}\text { Int J Clin Pedia- } \\
\text { tr Dent 2014; } \\
\text { 7(3):149-152. }\end{array}$ & $\begin{array}{l}\text { Stress Reduction through Audio } \\
\text { Distraction in Anxious Pediatric } \\
\text { Dental Patients: An Adjunctive } \\
\text { Clinical Study. }\end{array}$ & 2014 & India & $\begin{array}{l}60 \text { niños de } \\
6 \text { a } 12 \text { años } \\
\text { de edad }\end{array}$ \\
\hline $\begin{array}{l}\text { 2. Villasante V. } \\
\text { Rocío Padilla } \\
\text { C. Tania }{ }^{11}\end{array}$ & $\begin{array}{l}\text { Revista estomato- } \\
\text { lógica del altipla- } \\
\text { no.2014.1(1):27-34 }\end{array}$ & $\begin{array}{l}\text { Influencia de la técnica de } \\
\text { modelado con video en niños } \\
\text { no cooperadores durante el } \\
\text { tratamiento dental }\end{array}$ & 2014 & Perú & $\begin{array}{l}40 \text { niños de } \\
5 \text { a } 7 \text { años } \\
\text { de edad }\end{array}$ \\
\hline $\begin{array}{l}\text { 3. Attar, R.H., } \\
\text { Baghdadi, } \\
\text { Z.D }{ }^{25} \text {. }\end{array}$ & $\begin{array}{l}\text { Eur Arch Pediatr } \\
\text { Dent. 2015, } 16 \text { (8): }\end{array}$ & $\begin{array}{l}\text { Comparative efficacy of } \\
\text { active and passive distraction } \\
\text { during restorative treatment in } \\
\text { children using an iPad versus } \\
\text { audiovisual eyeglasses: a } \\
\text { randomised controlled trial. }\end{array}$ & 2015 & $\begin{array}{l}\text { Arabia } \\
\text { Saudita }\end{array}$ & $\begin{array}{l}39 \text { niños } 6 \\
\text { años }\end{array}$ \\
\hline $\begin{array}{l}\text { 4. S. Nuvvula } \\
\text { S. Alahari, R. } \\
\text { Kamatham y } \\
\text { RR Challa }^{26}\end{array}$ & $\begin{array}{l}\text { Eur Arch Pediatr } \\
\text { Dent. 2015; 16: } \\
43 \text { - } 50\end{array}$ & $\begin{array}{l}\text { Effect of audiovisual distraction } \\
\text { with 3D video glasses on } \\
\text { dental anxiety of children } \\
\text { experiencing administration of } \\
\text { local analgesia: a randomised } \\
\text { clinical trial } \\
\end{array}$ & 2015 & India & $\begin{array}{l}90 \text { niños } \\
\text { entre } 7 \text { y } 10 \\
\text { años }\end{array}$ \\
\hline $\begin{array}{l}\text { 5. Navit S, Johri } \\
\text { N, Khan SA, } \\
\text { Singh RK, } \\
\text { Chadha D, } \\
\text { Navit P, Shar- } \\
\text { ma A, Bahu- } \\
\text { guna } \mathrm{R}^{27} \text {. } \\
\end{array}$ & $\begin{array}{l}\text { J Clin Diagn Res. } \\
\text { 2015. Dec; 9(12): } \\
\text { ZC05-9. }\end{array}$ & $\begin{array}{l}\text { Effectiveness and Comparison } \\
\text { of Various Audio Distraction } \\
\text { Aids in Management of } \\
\text { Anxious Dental Pediatric } \\
\text { Patients }\end{array}$ & 2015 & India & $\begin{array}{l}150 \text { niños } \\
\text { entre } 6 \text { y } 12 \\
\text { años }\end{array}$ \\
\hline $\begin{array}{l}\text { 6. Al-Khotani } \\
\text { A, Bello LA, } \\
\text { Christidis N. }{ }^{28}\end{array}$ & $\begin{array}{l}\text { Acta Odontol } \\
\text { Scand. 2016; } \\
4(6): 494-501\end{array}$ & $\begin{array}{l}\text { Effects of audiovisual } \\
\text { distraction on children's } \\
\text { behaviour during dental } \\
\text { treatment: a randomized } \\
\text { controlled clinical trial }\end{array}$ & 2016 & $\begin{array}{l}\text { Arabia } \\
\text { Saudita }\end{array}$ & $\begin{array}{l}56 \text { niños } \\
\text { entre } 7 \text { y } \\
9 \text { años de } \\
\text { edad }\end{array}$ \\
\hline $\begin{array}{l}\text { 7. Loayza Susa- } \\
\text { na, Azanza } \\
\text { Sofia }^{29}\end{array}$ & $\begin{array}{l}\text { Revista de } \\
\text { Odontopediatria } \\
\text { Latinoamericana. } \\
2017(7): 2\end{array}$ & $\begin{array}{l}\text { Eficacia de dos técnicas de } \\
\text { acondicionamiento para la } \\
\text { atención odontológica de niños } \\
\text { de } 6 \text { a } 10 \text { años de edad de } \\
\text { una escuela pública de Quito- } \\
\text { Ecuador }\end{array}$ & 2017 & Ecuador & $\begin{array}{l}\text { Niños de } \\
\text { 6-10 años }\end{array}$ \\
\hline $\begin{array}{l}\text { 8. Alarco-Cadi- } \\
\text { llo Lorena, } \\
\text { Casas Apayco } \\
\text { Leslie, Reyes } \\
\text { Bossio Mario, } \\
\text { Ramírez } \\
\text { Torres María } \\
\text { Cecilia }^{30}\end{array}$ & $\begin{array}{l}\text { Revista de } \\
\text { Odontopediatría } \\
\text { Latinoamericana. } \\
2017 ; 7(1)\end{array}$ & $\begin{array}{l}\text { Uso de dos técnicas } \\
\text { Alternativas de manejo de } \\
\text { conducta: musicoterapia y } \\
\text { distracción audiovisual, en el } \\
\text { control y manejo de ansiedad } \\
\text { en pacientes pediátricos de } 5 \text { a } \\
10 \text { años }\end{array}$ & 2017 & Perú & $\begin{array}{l}60 \text { niños de } \\
5 \text { a } 10 \text { años }\end{array}$ \\
\hline
\end{tabular}


Tabla 1: Caracteristicas de los estudios incluidos en la revisión de la literatura Efectividad de las técnicas de manejo conductual en odontopediatría. Cont. 2

\begin{tabular}{|c|c|c|c|c|c|}
\hline Autores & Fuente & Titulo & Año & País & Muestra \\
\hline $\begin{array}{l}\text { 9. Aruna Pras- } \\
\text { hanth Vi- } \\
\text { shwakarma, } \\
\text { Prashant } \\
\text { Arjun Bon- } \\
\text { darde, Sudha } \\
\text { Bhiman- } \\
\text { gouda Patil, } \\
\text { Arun Suresh } \\
\text { Dodama- } \\
\text { ni,Prashanth } \\
\text { Yachrappa } \\
\text { Vishwakar- } \\
\text { ma, Shoeb A } \\
\text { Mujawar }{ }^{31} \\
\end{array}$ & $\begin{array}{l}\text { J Indian Soc } \\
\text { Pedod Prev Dent. } \\
\text { 2017; 35(2):143- } \\
149 .\end{array}$ & $\begin{array}{l}\text { Effectiveness of two different } \\
\text { behavioral modification } \\
\text { techniques among 5-7-year- } \\
\text { old children: A randomized } \\
\text { controlled trial }\end{array}$ & 2017 & India & $\begin{array}{l}98 \text { Niños } \\
\text { de } 5 \text { a } 7 \\
\text { años de } \\
\text { edad }\end{array}$ \\
\hline $\begin{array}{l}\text { 10. Garro- } \\
\text { cho-Rangel } \\
\text { A,. Iba- } \\
\text { rra-Gutiérrez } \\
\text { E, Rosa- } \\
\text { les-Bérber M, } \\
\text { Esquivel-Her- } \\
\text { nández R.,. } \\
\text { Esparza-Vi- } \\
\text { llalpando V, } \\
\text { Pozos-Guillén } \\
\text { A }^{32}\end{array}$ & $\begin{array}{l}\text { European Journal } \\
\text { of Paediatric } \\
\text { Dentistr.2018; } \\
\text { 19(1):74-79 }\end{array}$ & $\begin{array}{l}\text { A video eyeglasses/ earphones } \\
\text { system as distracting method } \\
\text { during dental treatment } \\
\text { in children: A crossover } \\
\text { randomised and controlled } \\
\text { clinical trial }\end{array}$ & 2018 & Mexico & $\begin{array}{l}40 \text { niños en } \\
\text { edades de } 5 \\
\text { a } 8 \text { años }\end{array}$ \\
\hline $\begin{array}{l}\text { 11. Khandelwal } \\
\text { D, Kalra N, } \\
\text { Tyagi R, Kha- } \\
\text { tri A, Gupta } \\
\mathrm{K}^{33}\end{array}$ & $\begin{array}{l}\text { J Contemp Dent } \\
\text { Pract. 2018, } \\
\text { 1;19(9): 1058-1064 }\end{array}$ & $\begin{array}{l}\text { Control of Anxiety in Pediatric } \\
\text { Patients using "Tell Show } \\
\text { Do" Method and Audiovisual } \\
\text { Distraction }\end{array}$ & 2018 & India & 400 niños \\
\hline $\begin{array}{l}\text { 12. Radhakrishna } \\
\text { S, Srinivasan } \\
\text { I, Setty JV1, } \\
\text { D R MK, } \\
\text { Melwani A, } \\
\text { Hegde KM }{ }^{34} \text {. } \\
\end{array}$ & $\begin{array}{l}\text { J Dent Anesth } \\
\text { Pain Med. } 2019 \\
\text { Feb; 19(1):29-36. }\end{array}$ & $\begin{array}{l}\text { Comparison of three behavior } \\
\text { modification techniques } \\
\text { for management of anxious } \\
\text { children aged } 4-8 \text { years. }\end{array}$ & 2019 & India & $\begin{array}{l}60 \text { niños } \\
\text { entre } 4-8 \\
\text { años de } \\
\text { edad }\end{array}$ \\
\hline $\begin{array}{l}\text { 13. Valenzuela } \\
\text { Muñoz A, } \\
\text { Valenzuela } \\
\text { Ramos MR, } \\
\text { Valenzuela } \\
\text { R }^{35}\end{array}$ & $\begin{array}{l}\text { Av } \\
\text { Odontoestomatol } \\
2019 ; .35(1): 27-31\end{array}$ & $\begin{array}{l}\text { Técnica de distracción } \\
\text { audiovisual para el control de } \\
\text { la ansiedad en niño }\end{array}$ & 2019 & España & $\begin{array}{l}400 \text { niños } \\
\text { de } 6 \text { a } 8 \\
\text { años de } \\
\text { edad }\end{array}$ \\
\hline
\end{tabular}


Tabla 2-1: Resultados de la revisión de los Métodos en los estudios sobre Efectividad de las Técnicas de manejo conductual en Odontopediatría

\begin{tabular}{|c|c|}
\hline Autores & Método \\
\hline $\begin{array}{l}\text { 1. Singh D, } \\
\text { Samadi et al } \\
(2014)^{24}\end{array}$ & $\begin{array}{l}\text { Niños sin experiencia dental. Se dividieron al azar en } 2 \text { grupos de } 30 \text { niños cada uno, el grupo } \\
\text { experimental escucho por auriculares, audios con música de su selección durante el procedi- } \\
\text { miento odontológico. El nivel de ansiedad se estableció usando la prueba de imagen de Venham. } \\
\text { La frecuencia del pulso y la saturación de oxígeno fueron medidas utilizando un oxímetro de } \\
\text { pulso. }\end{array}$ \\
\hline $\begin{array}{l}\text { 2. Villasante } \\
\text { y Padilla. } \\
(2014)^{11}\end{array}$ & $\begin{array}{l}\text { Estudio experimental. Con muestra aleatoria dividida en } 2 \text { grupos. El grupo experimental obser- } \\
\text { vó individualmente, durante } 10 \text { minutos un video con música e imágenes de dos niños de ambos } \\
\text { sexos con conductas cooperadoras durante la atención odontológica. El grupo control, observó, un } \\
\text { programa de un canal infantil. Ambos grupos fueron evaluados por un Odontopediatra antes de } \\
\text { la intervención y posterior a la misma para establecer según escala de Frankl si hubo modificación } \\
\text { de conducta. }\end{array}$ \\
\hline $\begin{array}{l}\text { 3. Attary Bagh- } \\
\text { dadi, }(2015)^{25}\end{array}$ & $\begin{array}{l}\text { Estudio experimental aleatorio. Evaluó dolor y frecuencias cardíacas de un grupo de exposición } \\
\text { (tratamiento con la ayuda de un iPad) y el grupo control (tratamiento con la ayuda de gafas AV) } \\
\text { se compararon ambos grupos. }\end{array}$ \\
\hline $\begin{array}{l}\text { 4. S. Nuvvula S. } \\
\text { Alahari et al } \\
(2015)^{26}\end{array}$ & $\begin{array}{l}90 \text { niños divididos aleatoriamente en } 3 \text { grupos; grupo de distracción de control (técnicas de orien- } \\
\text { tación del comportamiento sin distracción), audio (técnicas básicas más música) y AV (técnicas } \\
\text { básicas más AV 3D). La ansiedad se evaluó mediante autoinforme, pulso, y comportamiento uti- } \\
\text { lizando la escala de calificación de comportamiento de Franmkl modificado de Wright y la escala } \\
\text { de Houpt y preferencias de los niños. }\end{array}$ \\
\hline $\begin{array}{l}\text { 5. Navit S et al } \\
(2015)^{27}\end{array}$ & $\begin{array}{l}\text { Estudio experimental. Asignación aleatoria de } 30 \text { niños a cinco grupos. El grupo experimental } \\
\text { se subdividió en } 4 \text { subgrupos, incluyendo } 30 \text { niños en cada uno: SubGrupo } 1 \text { - música instru- } \\
\text { mental, SubGrupo } 2 \text { - música de canciones infantiles, SubGrupo } 3 \text { canciones de películas. Sub- } \\
\text { Grupo 4: cuentos infantiles y populares en audio. Cada niño tuvo } 4 \text { visitas al odontólogo: visita } \\
\text { de detección, de profilaxis oral, de preparación de la cavidad y la restauración y procedimientos } \\
\text { realizados bajo anestesia local, como extracciones, pulpotomías y pulpectomías. La Evaluación } \\
\text { de la ansiedad se efectuó mediante prueba de imagen de Venham (VPT), escala de calificación de } \\
\text { ansiedad clínica de Venham (VCRS) y Oxímetro de pulso de dedo (NL-50D).Grupo control: niños } \\
\text { tratados bajo una atención dental normal. }\end{array}$ \\
\hline $\begin{array}{l}\text { 6. Al-Khotani et } \\
\text { al }(2016)^{28}\end{array}$ & $\begin{array}{l}\text { Ensayo aleatorizado controlado. Los pacientes se dividieron aleatoriamente en dos grupos: Expe- } \\
\text { rimental con distracción (grupo AV), se usó el i -theatre }{ }^{\mathrm{TM}} \text { que son anteojos que se conectan } \\
\text { a varios dispositivos como reproductores de DVD, y sistemas de juegos. Al grupo control no se } \\
\text { le suministro ningún tipo de distracción. La respuesta del niño al estrés dental en ambos grupos } \\
\text { se evaluó mediante una combinación de cinco medidas (1) el FIS para la ansiedad dental, (2) el } \\
\text { MVARS que es una escala de } 6 \text { categorías y cada una describe el estado del paciente en el sillón } \\
\text { dental cuando se realiza un procedimiento específico; (3) la presión arterial sistólica (s-BP); (4) la } \\
\text { presión arterial diastólica (d-BP); (5) el Pulso. Los valores obtenidos para FIS, MVARS, BP y PR se } \\
\text { promediaron para producir el valor medio de la visita de restauración. }\end{array}$ \\
\hline $\begin{array}{l}\text { 7. Loayza Susana, } \\
\text { Azanza Sofia. } \\
(2017)^{29}\end{array}$ & $\begin{array}{l}\text { Los niños fueron asignados aleatoriamente en dos grupos }(\mathrm{N}=20) \text {. Se aplicó técnica de acondi- } \\
\text { cionamiento a cada niño de acuerdo a cada grupo. El comportamiento se valoró con la escala de } \\
\text { Franmkl (definitivamente positivo y definitivamente negativo). Se determinó comportamiento } \\
\text { inicial del niño basado en sus expresiones faciales y en su forma de comportarse. Se proyectó un } \\
\text { video en Youtube (Cepillo mis dientes).Técnica de acondicionamiento distracción visual y auditi- } \\
\text { va. Después se aplicó tratamiento sin utilización de instrumental rotatorio o manual. Finalmente } \\
\text { se evaluó el comportamiento. }\end{array}$ \\
\hline $\begin{array}{l}\text { 8. Alarco-Cadillo } \\
\text { et al }(2016)^{30}\end{array}$ & $\begin{array}{l}\text { Se conformaron } 3 \text { grupos de forma aleatoria. Grupo control: los niños no recibieron ningún tipo } \\
\text { de técnica de musicoterapia o distracción audiovisual .El manejo de conducta se realizó con las } \\
\text { técnicas decir - mostrar - hacer y manejo de la contingencia. El 2do grupo (musicoterapia) utilizó } \\
\text { dos reproductores MP3 con música instrumental, el tercer grupo (Grupo distracción audiovisual) } \\
\text { uso lentes de realidad virtual Google Cardboard TM, con cuatro películas animadas de libre } \\
\text { elección, y audífonos. Para realizar la evaluación de la ansiedad pre y post-operatoria se utilizó el } \\
\text { Test de Dibujos de Venham y para la evaluación durante el tratamiento dental se utilizó la Escala } \\
\text { de Ansiedad de Venham. }\end{array}$ \\
\hline
\end{tabular}


Tabla 2-2: Resultados de la revisión de los Métodos en los estudios sobre Efectividad de las Técnicas de manejo conductual en Odontopediatría.

\begin{tabular}{|c|c|}
\hline Autores & Método \\
\hline $\begin{array}{l}\text { 9. Aruna } \\
\text { Prashanth } \\
\text { Vishwakarma } \\
\text { et al }(2017)^{31}\end{array}$ & $\begin{array}{l}98 \text { niños asignados al azar en dos grupos. Grupo I: los niños fueron condicionados para recibir } \\
\text { procedimientos dentales usando modelado en vivo seguido de profilaxis dental. Grupo II: técnica } \\
\text { de decir-jugar-hacer con objetos dentales de juegos seguido de profilaxis dental. Después de } 7 \text { días } \\
\text { los niños de ambos grupos recibieron tratamiento restaurador. Se evaluó frecuencia cardiaca. Se } \\
\text { aplicó escala de imagen facial(FIS) y el índice de Venham; antes de la intervención, después de } \\
\text { la intervención y durante el procedimiento para establecer el comportamiento. }\end{array}$ \\
\hline $\begin{array}{l}\text { 10. Garro- } \\
\text { cho-Rangel et } \\
\text { al }(2018)^{32}\end{array}$ & $\begin{array}{l}\text { Pacientes sin experiencia dental previa. Los niños tenían un nivel I o II de cooperación en la } \\
\text { escala de Frankel evaluados en la 1era sesión dental. Se conformaron } 2 \text { grupos: Experimental que } \\
\text { usó lentes de video (China-Visión)TM con auriculares y películas durante la sesión dental. El } \\
\text { grupo control no uso lentes de video. Se utilizó la escala de dolor flacc. Efectuaron mediciones } \\
\text { de frecuencia cardiaca y oxigeno durante } 4 \text { fases de tratamiento: Explicación de procedimiento y } \\
\text { exhibición de instrumentos, inyección de anestesia local, colocación de presas de goma, y pieza } \\
\text { de mano de alta velocidad. Se aplicaron otras técnicas de comportamiento: decir-mostrar-hacer y } \\
\text { comunicación verbal. }\end{array}$ \\
\hline $\begin{array}{l}\text { 11. Khandelwal et } \\
\text { al }(2018)^{33}\end{array}$ & $\begin{array}{l}400 \text { pacientes que acudieron para su primera visita dental. La ansiedad se registró usando la esca- } \\
\text { la de imagen facial (FIS), la prueba de imagen de Venham (VPT), la presión arterial, la frecuencia } \\
\text { del pulso (PR) y la saturación de oxígeno (SpO2) en diferentes etapas de la visita. Los pacientes } \\
\text { que acudieron a la primera visita dental fueron sometidos a un tratamiento restaurador con el } \\
\text { método Tell show do (TSD) y distracción audiovisual (AVD). }\end{array}$ \\
\hline $\begin{array}{l}\text { 12. Radhakrishna } \\
\text { et al }(2019)^{34}\end{array}$ & $\begin{array}{l}\text { Niños con puntaje de calificación de comportamiento de Frankl de } 2 \text { o 3, que requerían restau- } \\
\text { raciones de cavidades de Clase I y II se dividieron en tres grupos. Grupo 1: Tell-Show-Play-doh; } \\
\text { Grupo 2: juego de dentista para teléfonos inteligentes; y Grupo 3: técnica de decir-mostrar-hacer. } \\
\text { La frecuencia del pulso, la Escala de imagen facial (FIS), la escala de calificación de comporta- } \\
\text { miento de Frankl y las escalas de comportamiento FLACC (Rostro, Pierna, Actividad, Llorar, } \\
\text { Consolabilidad) se usaron para evaluar comportamiento. }\end{array}$ \\
\hline $\begin{array}{l}\text { 13. Valenzuela et } \\
\text { al }(2019)^{35}\end{array}$ & $\begin{array}{l}\text { Se seleccionaron niños sistémicamente sanos, con comportamiento levemente negativo, según } \\
\text { escala Frankl.A todos los niños se les aplicó la técnica de distracción audiovisual que consistía } \\
\text { en videos a través de anteojos de realidad virtual. Esta técnica se realizó durante la ejecución de } \\
\text { tratamientos pulpares El nivel de ansiedad se midió con la Escala de Imagen Facial (FIS). }\end{array}$ \\
\hline
\end{tabular}

Tabla 3-1: Resultados de los hallazgos en los estudios de la revisión sobre Efectividad de las Técnicas de manejo conductual en Odontopediatría

\begin{tabular}{|c|c|}
\hline Autores & Resultados \\
\hline $\begin{array}{l}\text { 1. Singh D, } \\
\text { Samadi et al } \\
(2014)^{24}\end{array}$ & $\begin{array}{l}\text { En el grupo experimental, se observó un cambio de }-13,7 \pm 12,8 \mathrm{lpm} \text { en la frecuencia del pulso, } \\
-3,4 \pm 5,7 \mathrm{~mm} \mathrm{Hg} \text { en PAS, }-1,5 \pm 7,1 \mathrm{~mm} \mathrm{Hg} \text { en PAD, un aumento de } 2(6,7 \%) \text { casos con saturación de } \\
\text { oxígeno }<95 \% \text { y Se observó un cambio medio de }-3,00 \pm 2,15 \text { en las puntuaciones de VPT en compa- } \\
\text { ración con el valor inicial. Todos los otros cambios fueron estadísticamente significativos ( }<<0,05) \text {. } \\
\text { En el grupo control, un cambio de } 5,0 \pm 17,4 \text { en la frecuencia del pulso, } 3,8 \pm 6,7 \mathrm{~mm} \mathrm{Hg} \text { en PAS, } \\
2,2 \pm 7,7 \mathrm{rnm} \mathrm{Hg} \text { en PAD, reducción absoluta en el número de casos con saturación de } \mathrm{O}_{2}<95 \% \text { y un } \\
\text { cambio promedio de } 1,90 \text { + Se observaron } 1,32 \text { en las puntuaciones de VPT en comparación con el } \\
\text { valor inicial. El cambio en la frecuencia del pulso, la PAD y la saturación de oxígeno no fue estadís- } \\
\text { ticamente significativo. La distracción con audio disminuyó la ansiedad en los pacientes, además, } \\
\text { los niños tuvieron respuesta positiva a las presentaciones musicales y quisieron escucharlos en sus } \\
\text { visitas posteriores. }\end{array}$ \\
\hline
\end{tabular}


Tabla 3-2: Resultados de los hallazgos en los estudios de la revisión sobre Efectividad de las Técnicas de manejo conductual en Odontopediatría.

\begin{tabular}{|c|c|}
\hline Autores & Resultados \\
\hline $\begin{array}{l}\text { 2. Villasante } \\
\text { y Padilla. } \\
(2014)^{11}\end{array}$ & $\begin{array}{l}\text { En el grupo experimental se observó que los niños de } 5 \text { a } 7 \text { años de edad con conducta negativa } \\
(75 \%) \text { y definitivamente negativa }(25 \%) \text {, a la aplicación del video preoperatorio modificaron su } \\
\text { conducta a positiva }(60 \%) \text { y definitivamente positiva }(20 \%) \text { se encontró diferencia estadísticamente } \\
\text { significativa ( }>>0,05) \text { en relación a la atención odontológica. En el grupo control no observó el video } \\
\text { preoperatorio. Los autores concluyeron que la técnica no farmacológica de modelado con video } \\
\text { preoperatorio, influyó en el cambio de conducta de los niños no cooperadores. }\end{array}$ \\
\hline $\begin{array}{l}\text { 3. Attary Bagh- } \\
\text { dadi, }(2015)^{25}\end{array}$ & $\begin{array}{l}\text { Los niños con lentes AV presentaron puntuaciones mas altas de dolor y comportamiento que los } \\
\text { que usaron iPad. Los resultados del dolor mostraron diferencias significativas entre las dos técnicas } \\
\text { de distracción durante la administración de anestesia local }(\mathrm{p} 0,076) \text { y la eliminación de caries (p } \\
0,071 \text { ). se reportó una diferencia significativa entre las dos técnicas solo durante la administración } \\
\text { de anestesia local (p } 0,017) \text {, a favor del iPad. Las frecuencias cardiacas promedio durante los inter- } \\
\text { valos de tratamientos fueron mas bajas entre el grupo significativamente mas cortas para iPad. La } \\
\text { distracción activa con un iPad mostro un mejor rendimiento que la distracción pasiva con lentes AV. }\end{array}$ \\
\hline $\begin{array}{l}\text { 4. S. Nuvvula S. } \\
\text { Alahari et al } \\
(2015)^{26}\end{array}$ & $\begin{array}{l}\text { Hubo reducción altamente significativa en la ansiedad del grupo audiovisual del grupo según lo } \\
\text { informado por los valores de MCDAS ( })(p<0,001) \text { y la escala de Houpt }(p=0,003) \text {; mientras que la } \\
\text { frecuencia del pulso mostro un aumento estadisticamente significativo }(p<0,001) \text { en los tres grupos, } \\
\text { independientemente de la distración. Las preferencias de los niños también afirmaron el uso de } \\
\text { lentes de video 3D. }\end{array}$ \\
\hline $\begin{array}{l}\text { 5. Navit S et al } \\
(2015)^{27}\end{array}$ & $\begin{array}{l}\text { Al comparar la ansiedad autoinformada entre el grupo de control y los grupos de distracción por } \\
\text { audio, no se observaron diferencias estadísticamente significativas en la puntuación de VPT en } \\
\text { todas las visitas. } \\
\text { Cuando se midió la ansiedad con la escala de calificación clínica de Venham, no se encontraron } \\
\text { diferencias significativas entre los diferentes grupos. Al comparar los grupos control con el de } \\
\text { audio, el aumento del nivel de ansiedad para los grupos de audio fue menor que el de rimas } \\
\text { infantiles, canciones e historias. No se observaron diferencias significativas en las puntuaciones de } \\
\text { VPT y VCRS entre todos los grupos. }\end{array}$ \\
\hline $\begin{array}{l}\text { 6. Al-Khotani et } \\
\text { al }(2016)^{28}\end{array}$ & $\begin{array}{l}\text { De acuerdo con la escala FIS, ninguno de los pacientes incluidos informó ansiedad al inicio del } \\
\text { estudio: El 57\% dijo que estaba "muy feliz" y el } 43 \% \text { dijo que estaba "feliz" en el grupo CTR. } \\
\text { Mientras que el } 43 \% \text { dijo que estaba "muy feliz", y el } 57 \% \text { dijo que estaba "feliz" en el grupo AV. Al } \\
\text { analizar la ansiedad clínica con MVARS, hubo una reducción significativa de la ansiedad clínica } \\
\text { durante todo el procedimiento de restauración, incluida la inyección con anestesia local, en el gru- } \\
\text { po AV (p = 0,04), donde fue de } 0,71 \text { antes del procedimiento de restauración y de } 0,25 \text { en el fin del } \\
\text { procedimiento. Esta reducción significativa no se encontró en el grupo CTR (p>0.05), Los niños } \\
\text { que utilizan la distracción AV con la pantalla de anteojos y gafas durante el tratamiento dental } \\
\text { restaurativo informaron menos angustia durante el procedimiento que los que no lo tenían, y } \\
\text { mostraron una respuesta más positiva después de la inyección con anestesia local. }\end{array}$ \\
\hline $\begin{array}{l}\text { 7. Loayza Susana, } \\
\text { Azanza Sofia. } \\
(2017)^{29}\end{array}$ & $\begin{array}{l}\text { De los } 40 \text { niños evaluados el } 65 \% \text { de valoró como definitivamente negativo su percepción inicial } \\
\text { del tratamiento odontológi } \neg \text { co, y 35\% señaló que la experiencia fue definitivamente positiva. } \\
\text { Después de aplicar la técnica decir mostrar y hacer vemos que de los } 40 \text { niños evaluados; el } 10 \\
\% \text { la valoró como definiti } \neg \text { vamente negativo y el } 90 \% \text { como definitivamente positivo. Al aplicar } \\
\text { la técnica de distrac } \neg \text { ción visual auditiva el } 25 \% \text { señaló que definitiv-amente fue negativo y } 80 \% \\
\text { que fue definitivamente positivo. Al comparar los valores obtenidos entre las dos técnicas, no se } \\
\text { encontró diferencia significativa p= }(0,41) \text {. }\end{array}$ \\
\hline $\begin{array}{l}\text { 8. Alarco-Cadillo } \\
\text { et al }(2016)^{30}\end{array}$ & $\begin{array}{l}\text { El promedio de puntaje obtenido de la consulta dental con el Test de Venham para el grupo control } \\
\text { fue de } 2 \text {; para el grupo musicoterapia fue de } 1,4 \text { y de } 1,45 \text { para el grupo audiovisual; el promedio } \\
\text { de la escala de ansiedad durante la consulta para el grupo control fue de } 1,4 \text { y de } 0,2 \text { para musicote- } \\
\text { rapia y audiovisual. El puntaje después de la consulta dental para el grupo control fue de } 1,95 \text { para } \\
\text { musicoterapia fue de } 0,45 \text { y para el grupo audio visual fue de } 0.3 \text {. Se encontraron diferencias signi- } \\
\text { ficativas para la evaluación de la ansiedad con la escala de Venham aplicada durante el tratamiento } \\
\text { dental entre los } 3 \text { grupos de estudio ( } \mathrm{p}=0,001) \text {. }\end{array}$ \\
\hline
\end{tabular}


Tabla 3-3: Resultados de los hallazgos en los estudios de la revisión sobre Efectividad de las Técnicas de manejo conductual en Odontopediatría.

\begin{tabular}{|c|c|}
\hline Autores & Resultados \\
\hline $\begin{array}{l}\text { 9. Aruna } \\
\text { Prashanth } \\
\text { Vishwakarma } \\
\text { et al }(2017)^{31}\end{array}$ & $\begin{array}{l}\text { Las puntaciones promedio de la frecuencia del pulso, FIS y la escala de Venham fueron significa- } \\
\text { tivamente mas bajas en los niños que recibieron intervención de decir - jugar - hacer que los que } \\
\text { recibieron intervención de modelado vivo. Según los autores la técnica decir - jugar - hacer es eficaz } \\
\text { para reducir el miedo y la ansiedad de los niños sobre el tratamiento dental, los niños disfrutaron } \\
\text { jugando con objetos dentales personalizados. Los autores concluyeron que la técnica decir - jugar - } \\
\text { hacer es una alternativa de modificación del comportamiento durante la consulta odontopediatrica. }\end{array}$ \\
\hline $\begin{array}{l}\text { 10. Garro- } \\
\text { cho-Rangel et } \\
\text { al }(2018)^{32}\end{array}$ & $\begin{array}{l}\text { No hubo diferencias significativas entre las intervenciones/ sesiones dentales (uso de lentes de vi- } \\
\text { deo de teatro privado virtual (Chinavisión®; Kowloon, Hong Kong, China), con auriculares, versus } \\
\text { sin uso de lentes de video durante las cuatro fases de tratamiento, en cualquiera de las evaluaciones } \\
\text { efectuadas. }\end{array}$ \\
\hline $\begin{array}{l}\text { 11. Khandelwal et } \\
\text { al }(2018)^{33}\end{array}$ & $\begin{array}{l}\text { El cambio en la puntuación media de FIS y VPT de la etapa } 1 \text { a etapa } 3 \text { mostró una tendencia cre- } \\
\text { ciente del grupo I al grupo IV decir- mostrar- hacer (DMH) + DA (distracción audiovisual). Esta } \\
\text { disminución de la ansiedad en los grupos II, III Y IV fue estadísticamente significativo }(\mathrm{p}<0,05) \\
\text { en comparación con el grupo I. En cambio, en la puntuación media del FIS y Venhman, desde la } \\
\text { etapa } 1 \text { a la etapa } 3 \text { mostro una tendencia creciente del grupo I al grupo IV (decir - mostrar - hacer } \\
\text { + distracción audiovisual). Esta disminución de la ansiedad mostró una significancia estadística } \\
\text { (p<0,05) cuando se comparó con el grupo I. El grupo IV (DMH+DA) mostró la máxima disminución } \\
\text { de ansiedad entre los grupos. Los resultados mostraron frecuencia de pulso medidas más bajas, } \\
\text { puntajes más bajos de FIS Y FLACC y un mayor porcentaje de niños con un puntaje de } 4 \text { en la escala } \\
\text { de Frankl cuando se les aplicó las técnicas de juego de dentistas decir - jugar - hacer con teléfono } \\
\text { inteligente. Los autores afirman que la distracción audiovisual es mas capaz de reducir la ansiedad } \\
\text { que la técnica decir - mostrar - hacer. }\end{array}$ \\
\hline $\begin{array}{l}\text { 12. Radhakrishna } \\
\text { et al }(2019)^{34}\end{array}$ & $\begin{array}{l}\text { Se obtuvo una diferencia estadísticamente significativa entre las puntuaciones medias de FIS en } \\
\text { las etapas } 2 \text { y } 4 \text { del procedimiento dental del Grupo } 1(\mathrm{p}=0,005) \text { y el Grupo } 2(\mathrm{p}=0,01) \text {, lo que indica } \\
\text { una mayor reducción de la ansiedad dental, en comparación con el Grupo } 3 \text {. Se obtuvo una dife- } \\
\text { rencia estadísticamente significativa entes las etapas } 3 \text { y } 4 \text { del procedimiento dental en el grupo } \\
\text { decir - mostrar - hacer ( } \mathrm{p}=0,005) \text {, lo que indica mayor reducción de la ansiedad dental entre todos } \\
\text { los grupos. Los puntajes de FLACC mostraron que mas pacientes reflejaron con el juego de dentista } \\
\text { con teléfonos inteligentes y en los grupos decir- mostrar- jugar en comparación con el grupo decir } \\
\text { - mostrar - hacer que fue estadísticamente significativo. }\end{array}$ \\
\hline $\begin{array}{l}\text { 13. Valenzuela et } \\
\text { al }(2019)^{35}\end{array}$ & $\begin{array}{l}\text { La ansiedad leve aumentó después de realizado el tratamiento de un } 18 \% \text { a un } 47 \% \text {. La ansiedad } \\
\text { moderada a severa disminuyó de } 16 \% \text { a } 3 \% \text {, se observa que ningún niño después de realizado el } \\
\text { tratamiento tiene ansiedad severa. Siendo estos resultados estadísticamente significativos. La ansie- } \\
\text { dad severa disminuyó de un } 11 \% \text { a un } 0 \% \text {. }\end{array}$ \\
\hline
\end{tabular}

\section{Discusión}

Esta revisión se centró en la evaluación de la efectividad de las técnicas del manejo de conductas en niños sistémicamente sanos, no cooperadores, en odontopediatría.

En los estudios analizados hubo gran homogeneidad en cuanto a la selección de las pruebas y escalas para medir los niveles de ansiedad en los niños al recibir tratamiento preventivo, curativo o restaurador, estas fueron: prueba Venham ${ }^{24,27,30,31}$ escala de imagen facial (FIS) ${ }^{28,31,33-35}$ y las respuestas fisiológicas como el ritmo cardiaco y la presión arterial $^{26-28}$ así como la saturación de oxigeno. ${ }^{24,27}$ Esto concuerda con diversas investigaciones que para determinar los niveles de ansiedad en los niños y adolescentes en la consulta de odontopediatría han utilizado estos instrumentos y escalas que han sido ampliamente probados y validados. ${ }^{36,37}$ 
Crego $e t a l^{38}$ señalan que la prevalencia de la ansiedad dental en los niños es difícil establecer, afirmando que las tasas varían dependiendo de las características del estudio y edades, y que existen cifras que las estiman desde 5,7 a $19,5 \%$ con una media general de $11,1 \%$ en niños de Europa. Estos autores plantean el llamado circuloviciosodela conexiónenfermedades orales-ansiedad dental señalando que la ansiedad y miedo dental disminuyen las frecuencias de las visitas a los servicios de atención odontológica; esto redunda en una mayor presencia de enfermedades orales que al hacerse severas, requieren tratamientos e intervenciones más complejos, urgentes y costosos; hechos que acrecientan la ansiedad y miedo, por lo que las patologías bucales permanecen sin tratamiento lo que repercute en la salud bucal y en la calidad de vida.

Por ello se recomienda que en los estudios epidemiológicos que determinan la prevalencia de las enfermedades bucales, principalmente caries dental y enfermedad periodontal, considerados como problemas de salud pública por la OMS, se incluyan las variables fobias dentales, miedo y ansiedad.

En esta revisión el comportamiento de los niños fue determinado utilizando la escala Frankel, 29, 32, 34,35 Frankel modificado por Wright, escala de Houpt ${ }^{26}$ y la escala FLACC ${ }^{32,34}$. Casi todos los estudios evaluaron la aplicación de técnicas de distracción con diversos recursos entre ellos audios con música ${ }^{24,11,25}$, videos $^{11,28,29,32,34,35}$ tres (3) estudios aplicaron una variación de la técnica decir-mostrarhacer, por decir-jugar-hacer a través de juegos instalado en teléfonos denominados "inteligentes ${ }^{31,33}$ y juegos con objetos dentales personalizados. ${ }^{31}$
También hubo estudios que aplicaron combinaciones de los recursos música, videos, ${ }^{26,27,30}$ todo ello para efectuar comparaciones de la efectividad de una técnica de distracción respecto a otra, con un grupo control o con variaciones de la misma intervención. El objetivo es lograr que el paciente niño desvíe la atención ante lo que pueda ser percibido como un procedimiento desagradable, evitando así conductas negativas o de rechazo. ${ }^{19}$

Con el avance de la tecnología se empezó a enfatizar el uso de las técnicas audiovisuales durante la atención odontológica en niños, se han efectuado estudios que reportan disminución de los niveles de ansiedad y miedo asociados con el tratamiento dental, logrando así pacientes colaboradores. ${ }^{39}$

$\mathrm{Mann}^{40}$ sostiene que actualmente los niños hacen uso del lenguaje y de la tecnología mejor que los adultos, con mayor confianza y libertad y sin temor a equivocarse y que el mundo actual del niño es un mundo de fantasía y tecnología, por lo tanto el odontopediatría debe actualizarse constantemente en los conocimientos del mundo infanto-juvenil actual (música, video, juegos, películas, personajes, moda, vocabulario, etc.) para tender un puente de comunicación y respeto mutuo, obteniendo de esta manera, la colaboración del niño/adolescente.

Los estudios reportaron disminución de los niveles de ansiedad y miedo, frecuencias cardiacas, pulso y saturación de oxígeno en los niños, después de la aplicación de las técnicas de distracción, así mismo informaron que se produjeron cambios de conducta de pacientes ansiosos y negativos a pacientes positivos y colaboradores. 


\section{Conclusiones}

Los estudios de esta revisión mostraron una insuficiente calidad en los diseños de los ensayos aleatorios controlados, además de que la limitada cantidad de los mismos, no permiten emitir juicios respecto a la contundencia y solidez de la evidencia científica sobre la efectividad de las técnicas de manejo de la conducta en niños sistémicamente sanos, en la consulta odontopediátrica.

\section{Limitaciones}

Al considerar las potenciales limitaciones de este tra $\neg$ bajo, es necesario señalar los obstáculos para obtener la literatura científica en textos completos ya que al no contar con una fuente de financiamiento el acceso a la literatura científica se hizo algo restringido. Otro elemento fue circunscribir la búsqueda de los artículos a solo dos idiomas, español e inglés y en un periodo de cinco años; razones por la cual, a pesar de los esfuerzos por intentar presentar un panorama general en torno a la temática sobre la efectividad de las técnicas de manejo conductual en odontopediatría, esta sigue siendo, limitada lo que podría constituir un sesgo en los resultados de esta investigación.
Implicaciones para la práctica

Los odontopediatra deben seguir enfatizando la importancia de educar a padres, cuidadores y niños sobre la importancia del componente bucal de la salud, sus factores de riesgo y patologías más prevalentes a fin de generar conductas $\mathrm{y}$ actitudes positivas y sanas hacia la salud bucal.

Implicaciones para la investigación

Se requiere efectuar más investigaciones de tipo experimental que aumenten la rigurosidad de los diseños aleatorios, controlados, disminuyendo así los posibles errores de sesgos (selección, detección o notificación entre otros) y con una duración suficiente que permita demostrar efectivamente, con la aplicación de las diversas técnicas, los cambios en un aspecto tan complejo como el comportamiento y las actitudes de los niños hacia la atención odontológica.

\section{Conflicto de interés}

Los autores declaran no tener conflictos de interés, ni fuentes de financiamiento.

\section{Referencias bibliográficas}

1. Odabas M, Bodur H, Baris E, Demir C. Clinical, Radiographic, and Histopathologic Evaluation of Nd: YAG Laser Pulpotomy on Human Primary Teeth. J Endod [Internet]. 2007 [Consultado 2019 Jun 10]; 33: 415-21. Disponible en: https://www.jendodon.com/article/S0099-2399(06)01221-0/fulltext

2. IARC Classifies Formaldehyde As Carcinogenic To Humans. IARC Monogr Eval Carcinog Risks Hum [Internet]. 2006 [Consultado 2019 May 10]; 62:217-375 Disponible en: https://monographs.iarc.fr/wpcontent/uploads/2018/06/mono88.pdf

3. Zaror C, Vergara C, Díaz J, Aracena D. Pulpotomías con sulfato férrico y MTA en dientes primarios: serie de casos. Int. J. Odontostomat [Internet]. 2011 [Consultado 2019 Jun 2]; 5(1):77-82. Disponible en: https:// scielo.conicyt.cl/scielo.php?script=sci_arttext\&pid=S0718-381X2011000100012 
4. Srinivasan V, Patchett CL, Waterhouse PJ. Is there life after Buckley's Formocresol? Part I - A narrative review of alternative interventions and materials. Int J Paed Dent [Internet]. 2006 [Consultado 2019 Jun 10]; 16: 117-27. Disponible en: https://www.ncbi.nlm.nih.gov/pubmed/16430526

5. Assed S, Assed L, Nelson-Filho P. Pulpotomía en dientes temporales y permanentes jóvenes. En: Assed L, editor. Tratado de Odontopediatría. 1 ed. Colombia: Amolca; 2008. p. 571-611.

6. Biedma M, Solano B, García F, Mendoza A, Iglesias A. Clinical and radiographic evaluation of white MTA versus formocresol Pulpotomy: a 48-month follow-up study. Am J Dent [Internet]. 2017 [Consultado 2019 Jun 2]; 30(3):131-136. Disponible en: https://www.ncbi.nlm.nih.gov/pubmed/29178757

7. Valdivieso M, Huamán M. Diagnóstico y tratamiento pulpar. En: Castillo R Perona G, Kanashiro C, Perea M, Silva-Esteves F, editores. Estomatología Pediátrica. 1 ed. España:Ripano “Editorial Médico"; 2011. p. 174-99.

8. Rodríguez P; Bolaños V. Propiedades y Usos en Odontopediatria del MTA (Agregado de Trióxido Mineral) Odovtos - International Journal of Dental Sciences [Internet]. 2011 [Consultado 2019 Jun 2]; 13, 2011, pp. 65-70 Disponible en: https://revistas.ucr.ac.cr/index.php/Odontos/article/view/4772

9. Aguado J, Cruz I,Maroto M, Barbería E. (2009). Posibilidades terapéuticas del Agregado Trióxido Mineral (MTA) en odontopediatría. JADA [Internet]. 2009 [Consultado 2019 Jun 2]; 4. 185-193. Disponible en: https://www.researchgate.net/publication/257920006_Posibilidades_terapeuticas_del_Agregado_ Trioxido_Mineral_MTA_en_odontopediatria

10. Hincapié S, Valerio AL. Biodentine: una nueva propuesta en terapia pulpar. Univ Odontol. 2015 [Consultado 2019 May 10]; 34(73): 69-76. Disponible en: https://revistas.javeriana.edu.co/index.php/ revUnivOdontologica/article/view/16040

11. Caicedo R, Abbott P, Alongi D, Alarcon M. Clinical, radiographic and histological analysis of the effects of mineral trioxide aggregate used in direct pulp capping and pulpotomies of primary teeth. Austr Dent J [Internet]. 2006 [Consultado 2019 Jun 14]; 51(4): 297-305. Disponible en: https://www.ncbi.nlm.nih.gov/ pubmed/17256303

12. Higgins JPT, Green S (editors). Cochrane Handbook for Systematic Reviews of Interventions Version 5.1.0 The Cochrane Collaboration [Internet]. 2011 [Consultado 2019 May 14]. Disponible en: https://es.cochrane. org/sites/es.cochrane.org/files/public/uploads/manual_cochrane_510_web.pdf

13. Kitchenham B. Procedures for Performing Systematic Reviews. Department of Computer Science, Keele University Technical Report [Internet]. 2004 [Consultado 2019 May 12]; 33. Disponible en: www.inf.ufsc. br/ aldo.vw/kitchenham.pdf

14. Kitchenham B, Brereton P, Budgen D, Turner M, Bailey J, Linkman S. Systematic literature reviews in software engineering-A systematic literature review. Information and Software Technology [Internet]. 2009 [Consultado 2019 May 12]; 51. 7-15. Disponible en: https://www.sciencedirect.com/science/article/ abs/pii/S0950584908001390

15. Junqueira M, Cunha N, Caixeta, Marques N, Oliveira T, Moretti A, Cosme L, Sakai V. Clinical, Radiographic and Histological Evaluation of Primary Teeth Pulpotomy Using MTA And Ferric Sulfate. Braz. Dent. J [Internet]. 2018 [Consultado 2019 Jun 2]; 29(2): 159-165. Disponible en: http://www.scielo.br/scielo. php?script=sci_arttext\&pid=S0103-64402018000200159\&lng=en.

16. Guven Y, Aksakal S, Avcu N, Unsal G, Tuna E, Aktoren O. Success Rates of Pulpotomies in Primary Molars Using Calcium Silicate-Based Materials: A Randomized Control Trial. Biomed Res Int [Internet]. 2017 [Consultado 2019 Jun 2]; 2017. Disponible en: https://www.ncbi.nlm.nih.gov/pubmed/29226134

17. Goyal P, Pandit I, Gugnani N, Gupta M, Goel R, Gambhir R. Clinical and radiographic comparison of various medicaments used for pulpotomy in primary molars: A randomized clinical trial. Eur J Dent [Internet]. 2016 [Consultado 2019 Jun 2]; 10:315-20. Disponible en: https://www.ncbi.nlm.nih.gov/ pubmed/27403046

18. Yildiz E, Tosun G. Evaluation of formocresol, calcium hydroxide, ferric sulfate, and MTA primary molar pulpotomies. Eur J Dent [Internet]. 2014 [Consultado 2019 Jun 2]; 8:234-40. Disponible en: https://www. ncbi.nlm.nih.gov/pubmed/24966776

19. Biondi A, Cortese S, Ortolani A. Pulpotomías en molares primarios evaluación clínica, radiográfica de formocresol o trióxido mineral agregado. Revista de la Facultad de Odontología- Repositorio (UBA) [Internet]. 2008 [Consultado 2019 Jun 2]; 23:54-55. Disponible en: http://od.odontologia.uba.ar/ revista/2008vol23num54-55/art2.pdf

20. Noorollahian H. Comparison of mineral trioxide aggregate and formocresol as pulp medicaments for pulpotomies in primary molars. Br Dent J [Internet]. 2008 [Consultado 2019 Jun 2]; 204(11). Disponible en: https://www.ncbi.nlm.nih.gov/pubmed/18425074 
21. Hugar S, Deshpande S. Comparative investigation of clinical/radiographical signs of mineral trioxide aggregate and formocresol on pulpotomized primary molars. Contemporary clinical dentistry [Internet]. 2010 [Consultado 2019 Jun 2]; 1(3), 146-151. Disponible en: https://www.ncbi.nlm.nih.gov/pmc/articles/ PMC3220100/

22. Ansari G, Ranjpour M. Mineral trioxide aggregate and formocresol pulpotomy of primary teeth: a 2-year follow- up. International Endodontic Journal [Internet]. 2010 [Consultado 2019 Jun 2]; 43(5):413-8. Disponible en: https://www.ncbi.nlm.nih.gov/pubmed/20518934

23. Godhi B, Sood P, Sharma A. Effects of mineral trioxide aggregate and formocresol on vital pulp after pulpotomy of primary molars: An in vivo study. Contemporary clinical dentistry [Internet]. 2011 [Consultado 2019 Jun 2]; 2(4), 296- 301. Disponible en: https://www.ncbi.nlm.nih.gov/pubmed/22346156

24. Hugar S, Reddy R, Deshpande S, Shigli A, Gokhale N, Hugar S. In vivo Comparative Evaluation of Mineral Trioxide Aggregate and Formocresol Pulpotomy in Primary Molars: A 60-month Follow-up Study. Contemporary clinical dentistry [Internet]. 2017 [Consultado 2019 Jun 2]; 8(1), 122-127. Disponible en: https://www.ncbi.nlm.nih.gov/pmc/articles/PMC5426144/

25. Jayam C, Mitra M, Mishra J, Bhattacharya B, Jana B. Evaluation and comparison of white mineral trioxide aggregate and formocresol medicaments in primary tooth pulpotomy: clinical and radiographic study. J Indian Soc Pedod Prev Dent [Internet]. 2014 [Consultado 2019 Jun 2]; 32(1):13-8. Disponible en: https:// www.ncbi.nlm.nih.gov/pubmed/24531596

26. Yildirim C, Basak F, Akgun O, Polat G, Altun, C. Clinical and radiographic evaluation of the effectiveness of formocresol, mineral trioxide aggregate, portland cement, and enamel matrix derivative in primary teeth pulpotomies: a two year follow-up. J Clin Pediatr Dent [Internet]. 2016 [Consultado 2019 Jun 2]; 40(1):14-20. Disponible en: https://www.ncbi.nlm.nih.gov/pubmed/26696101

27. Olatosi $\mathrm{O}$, Sote E, Orenuga O. Effect of mineral trioxide aggregate and formocresol pulpotomy on vital primary teeth: a clinical and radiographic study. Niger J Clin Pract [Internet]. 2015 [Consultado 2019 Jun 2]; 18(2):292-6. Disponible en: https://www.ncbi.nlm.nih.gov/pubmed/25666010

28. Moretti A, Sakai V, Oliveira T, Fornetti A, Santos C, Machado M, Abdo R. The effectiveness of mineral trioxide aggregate, calcium hydroxide and formocresol for pulpotomies in primary teeth. Int Endod J [Internet.] 2008 [Consultado 2019 Jun 2]; 41(7):547-55. Disponible en: https://www.ncbi.nlm.nih.gov/ pubmed/18479381

29. De Vasconcelos B , Bernardes R , Cruz S, Duarte M , Padilha M , Bernardineli N , Garcia R , Bramante C, de Moraes I . Evaluation of $\mathrm{pH}$ and calcium ion release of new root-end filling materials. Oral Surg Oral Med Oral Pathol Oral Radiol Endod. [Internet]. 2009 [Consultado 2019 Jun 2]; 108(1):135-9. Disponible en: https://www.ncbi.nlm.nih.gov/pubmed/19451009

30. Vallejo E, Rosales J. Materiales dentales. En: Boj JR. Odontopediatría. La evolución del niño al adulto joven. Madrid: Ripano editorial médica; 2011. p. 195-210.

31. Patchett CL, Srinivasan V, Waterhouse PJ. Is there life after Buckley's formocresol? Part II - Development of a protocol for the management of extensive caries in the primary molar. Int J Paed Dent [Internet]. 2006 [Consultado 2019 Jun 2]; 16: 199-206 Disponible en: https://www.ncbi.nlm.nih.gov/pubmed/16643542

32. Gonzalez Rodriguez E., Ruiz Linares M. Diagnóstico y tratamiento en dentición temporal. En: Boj JR, Catalá M, García-Ballesta C, Mendoza A. Odontopediatría. 1ª ed. Barcelona: Masson; 2004 p.173-183.

33. Isassi $\mathrm{H}$, Mendoza M, Padilla C. Estudio clínico de pulpotomía con agregado trióxido mineral en 58 molares primarios. Medigraphic [Internet]. 2012 [Consultado 2019 Jun 10]; Vol. 37. Disponible en: https:// www.medigraphic.com/pdfs/oral/ora-2011/ora1137g.pdf

34. Castillo R, Perona G, Kanashiro C, Perea M, Silva F. ESTOMATOLOGÍA PEDIÁTRICA, edit. RIPANO Madrid 2010; (174-191).

Recibido: 02/02/20

Aceptado: 17/07/2020

Correspondencia: Miriam Ortega, correo: mfortegal38@hotmail.com 